\title{
Methylhydrazinium Lead Bromide: Noncentrosymmetric Three- Dimensional Perovskite with Exceptionally Large Framework Distortion and Green Photoluminescence
}

\author{
Mirosław Mączka,* Maciej Ptak, Anna Ga̧gor, Dagmara Stefańska, Jan K. Zarȩba, and Adam Sieradzki
}

Cite This: Chem. Mater. 2020, 32, 1667-1673

Read Online

ABSTRACT: Three-dimensional (3D) lead halide perovskites have emerged as a promising class of coordination polymers for solar cells, photodetectors, and light-emitting devices. These compounds thus far comprise methylammonium, formamidinium, or cesium as cations. In this work, we introduce a new methylhydrazinium $3 \mathrm{D}$ perovskite, $\mathrm{CH}_{3} \mathrm{NH}_{2} \mathrm{NH}_{2} \mathrm{PbBr}_{3}$, that crystallizes in the polar $P 2_{1}$ structure at room temperature and undergoes a phase transition to the cubic $\operatorname{Pm} \overline{3} m$ phase at $418 \mathrm{~K}$. This perovskite exhibits strong second-harmonic generation activity, features switchable dielectric behavior, thermochromism, and two-photon energy upconversion under $800 \mathrm{~nm}$ excitation.

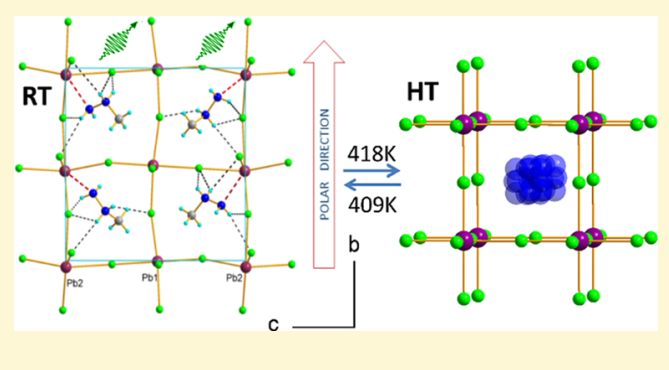

$3 \mathrm{D}$ hybrid perovskites of general formula $\mathrm{ABX}_{3}$, where $\mathrm{A}$ is an organic or alkali metal cation, $\mathrm{B}$ is a divalent cation (typically $\mathrm{Pb}^{2+}, \mathrm{Sn}^{2+}$, or $\mathrm{Ge}^{2+}$ ), and $\mathrm{X}=$ halogen, have emerged as a promising class of compounds for optoelectronic applications. ${ }^{1-4}$ The most commonly investigated perovskite, methylammonium $\left(\mathrm{MA}^{+}\right)$lead iodide, is one of the most promising photovoltaic materials with power conversion efficiency near $22 \% .^{2} 3 \mathrm{D}$ lead halides, especially $\mathrm{MAPbBr}_{3}$ and $\mathrm{FAPbBr}_{3}$ (FA = formamidinium $)$, are also attractive for light-emitting diodes ${ }^{4}$ and photodetectors. ${ }^{5}$ Furthermore, $\mathrm{MAPbBr}_{3}$ was shown to exhibit highly efficient multiphotonexcited upconversion PL that makes it suitable for many important applications such as in vivo imaging or photodynamic therapy. ${ }^{6}$ Excellent optoelectronic properties of 3D halides promoted broad interest in search for new analogues. According to the Goldschmidt tolerance factor $t, 3 \mathrm{D}$ perovskites should form for $0.8 \leq t \leq 1.0$. $^{1}$ For $t$ larger than 1.0 and smaller than 0.8 , lower-dimensional phases are usually formed that do not show optical properties suitable for solar cell applications. ${ }^{1,2,6}$ In the case of lead halides, the $\mathrm{PbX}_{3}{ }^{-}$ framework is predicted to accommodate only the smallest organic cations such as hydroxylammonium, $\mathrm{MA}^{+}$, hydrazinium, azetidinium, $\mathrm{FA}^{+}$, and imidazolium. ${ }^{1}$ Despite a large body of research in this field, 3D perovskite structures were reported only for lead halides comprising $\mathrm{MA}^{+}$and $\mathrm{FA}^{+}$ organic cations, ${ }^{1,2}$ while for the other small organic cations, non-perovskite or layered perovskite-like structures were formed. $^{7-10}$ This behavior is especially surprising for hydroxylammonium and hydrazinium cations, which have nearly the same size as $\mathrm{MA}^{+}$. It was argued that this behavior can be attributed to its ability to form strong hydrogen bonds (HBs) by these cations. ${ }^{7,8}$
HT phases of $\mathrm{MAPbX}_{3}$ and $\mathrm{FAPbX}_{3}$ adopt the cubic space group $P m \overline{3} m$, but they undergo successive phase transitions on cooling to tetragonal and then orthorhombic or trigonal phases. $^{2,11-13}$ Some studies suggested that the remarkable photovoltaic efficiencies of perovskites can be explained by the existence of ferroelectric order because ferroelectric polarization enables separation of electrons and holes. ${ }^{14}$ The presence of ferroelectricity also means that the material exhibits piezoelectricity and pyroelectricity, enabling construction of multifunctional devices. Thus, the existence or absence of ferroelectricity in lead halides has been a highly debated issue in recent years, but results obtained by different groups are contradictory. A necessary precondition for a material to be branded as ferroelectric is its noncentrosymmetric and polar structure. According to diffraction studies, 3D lead halide phases are centrosymmetric. ${ }^{11-13,15-17}$ However, diffraction methods might not detect very small deviations from the centrosymmetry, which contributes to the ongoing controversies in some cases. Indeed, ferroelectricity was suggested in the tetragonal phase of $\mathrm{MAPbI}_{3}$ by observation of ferroelectric domains ${ }^{18}$ and ferroelectric hysteresis, ${ }^{19}$ whereas theoretical models and pyrocurrent measurements suggested the nonferroelectric nature of all phases of $\mathrm{MAPbX}_{3}$ perovskites. ${ }^{20}$ Very recently, direct piezoelectric force microscopic measurements suggested the nonferroelectric nature of $\mathrm{MAPbI}_{3}{ }^{21}$

Received: December 20, 2019

Revised: January 23, 2020

Published: January 24, 2020 
Here, we report the synthesis and characterization of a new $3 \mathrm{D}$ perovskite comprising a methylhydrazinium $\left(\mathrm{MHy}^{+}\right)$ cation that has a significantly larger ionic radius (264 pm) than $\mathrm{FA}^{+}(253 \mathrm{pm}){ }^{1,22}$ Thus the calculated tolerance factor for $\mathrm{MHyPbBr}_{3}$ is 1.03 , that is, larger than 1 . We will show that unlike archetypal $\mathrm{MAPbX}_{3}$ and $\mathrm{FAPbX}_{3}$ analogues, the RT structure of this perovskite is strongly noncentrosymmetric.

Crystals of $\mathrm{MHyPbBr}_{3}$ were obtained at RT from $\mathrm{HBr}$ acid by antisolvent vapor-assisted crystallization. A good match of their powder XRD pattern with the calculated one (Figure S1) confirmed the bulk phase purity. $\mathrm{MHyPbBr}_{3}$ shows no sign of decomposition for at least 8 months when kept in a desiccator (Figure S1).

The DSC measurements show the presence of one heat anomaly at $T_{\mathrm{c}}=418 \mathrm{~K}(409 \mathrm{~K})$ during heating (cooling), respectively (Figure 1 and Figure S2). The strongly symmetric
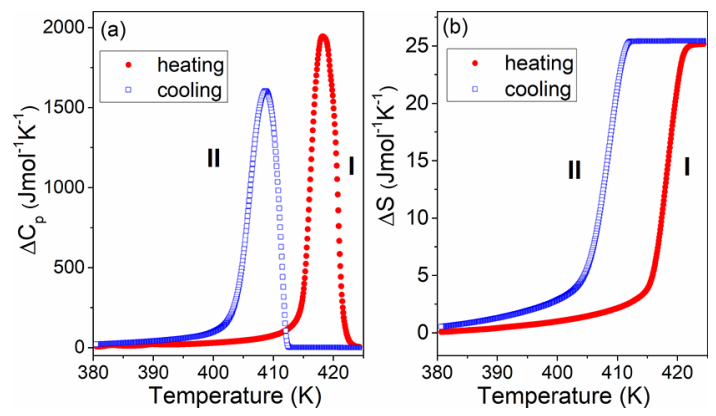

Figure 1. Change in (a) $C_{p}$ and (b) $S$ related to the phase transition in the heating (red) and cooling (blue) runs.

shape of this anomaly, sharp change of entropy, and large thermal hysteresis all point to the first-order character of the phase transition. The associated changes in enthalpy $\Delta H$ and entropy $\Delta S$ are estimated to be $\sim 9.9 \mathrm{~kJ} \mathrm{~mol}^{-1}$ and $\sim 25.3 \mathrm{~J}$ $\mathrm{mol}^{-1} \mathrm{~K}^{-1}$, respectively. For an order-disorder transition, $\Delta S$ $=R \ln (N)$ where $R$ is the gas constant and $N$ is the ratio of the number of configurations in the disordered and ordered phases. The estimated $N$ is 20.9 , indicating that the HT phase I of $\mathrm{MHyPbBr}_{3}$ is highly disordered.

The thermogravimetric plot indicates that $\mathrm{MHyPbBr}_{3}$ starts to decompose at $513 \mathrm{~K}$ (Figure S3). In this plot, a weight loss of $25.3 \%$ takes place between 513 and $675 \mathrm{~K}$, corresponding to the release of methylhydrazinium bromide (the calculated value is $25.7 \%$ ). On further heating, $\mathrm{PbBr}_{2}$ starts to sublimate and this process ends near $950 \mathrm{~K}$.

The HT phase $I$ is isostructural to $\mathrm{FAPbBr}_{3}$ and $\mathrm{MAPbBr}$ analogues. ${ }^{2,11,13}$ The perovskite substructure of the $P m \overline{3} m$ space group is built of ideal $\mathrm{PbBr}_{6}$ octahedra of $\mathrm{O}_{h}$ symmetry. All $\mathrm{Pb}-\mathrm{Br}$ distances are the same (3.018(1) $\AA$ at $430 \mathrm{~K})$, all $\mathrm{Br}-\mathrm{Pb}-\mathrm{Br}_{\text {cis }}$ angles are equal to $90^{\circ}$, whereas all $\mathrm{Br}-\mathrm{Pb}-\mathrm{Br}_{\text {trans }}$ and bridging $\mathrm{Br}-\mathrm{Pb}-\mathrm{Br}$ angles equal $180^{\circ}$ (Tables $\mathrm{S} 1$ and $\mathrm{S} 2$ ). Accordingly, bond length distortion and angle variance are equal $0 . \mathrm{MHy}^{+}$cations rotate freely within perovskite cavities (Figure S4).

The phase transition to phase II has order-disorder as well as a strong distortive character. The $\mathrm{MHy}^{+}$cations are ordered and all stack with $\mathrm{CH}_{3}$ groups toward the perovskite [100] layer built of $\mathrm{Pb}(1) \mathrm{Br}_{6}$ octahedra and with the $\mathrm{NH}_{2}{ }^{+}$group directed toward the neighboring layer consisting of $\mathrm{Pb}(2) \mathrm{Br}_{6}$. $\mathrm{NH}_{2}-\mathrm{NH}_{2}{ }^{+}$donor centers interact with bromine acceptors via $\mathrm{N}-\mathrm{H} \cdots \mathrm{Br}$ hydrogen bonds (HBs), see Table S3, whereas $\mathrm{Pb}(2)-\mathrm{NH}_{2}{ }^{+}$distances of 2.915(1) and 3.044(1) A fall within the maximum range of $\mathrm{Pb}-\mathrm{NH}_{2}$ coordinate bonds, see the histogram in Figure S5. All this brings massive distortion of the perovskite framework, especially of the part that surrounds the $\mathrm{NH}_{2}-\mathrm{NH}_{2}^{+}$groups. The symmetry is reduced to monoclinic $P 2_{1}$, and the unit cell parameters double in the $b$ and $c$ directions to accommodate the monoclinic distortion and differentiate neighboring [100] layers into two: less distorted, built of $\mathrm{Pb}(1) \mathrm{Br}_{6}$, and greatly deformed, consisting of $\mathrm{Pb}(2) \mathrm{Br}_{6}$. The tilts of $\mathrm{PbBr}_{6}$ octahedra can be described as $a^{+} b^{0} c^{0}$, according to Glazer's notation. ${ }^{23}$ The same type of tilting was reported for $\mathrm{FAPbX}_{3}$ perovskites. ${ }^{12,17}$ Thus, the phase transition involves condensation of one of the triply degenerate octahedral rotation of $\mathrm{M}_{3}^{+}$symmetry. the polar nature of II and ordering of $\mathrm{MHy}^{+}$indicate that the order parameter corresponds also to displacement of organic cations of $\Gamma_{4}^{-}$symmetry and a mode involving collective tumbling of $\mathrm{MHy}^{+}$. However, very low symmetry of II implies also condensation of a few other optical phonons from $\Gamma, X$, and $M$ points of the Brillouin zone. Figure 2 illustrates the (a)

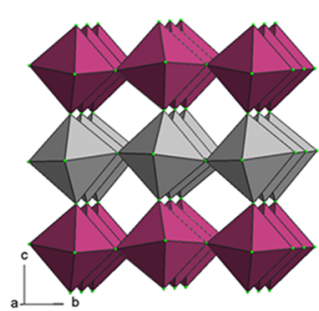

(b)

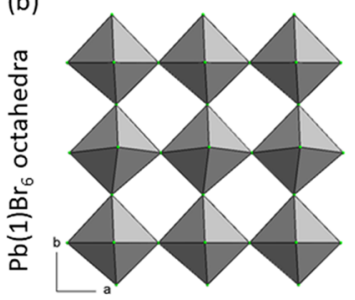

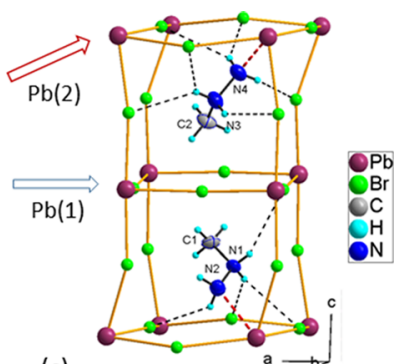

(c)

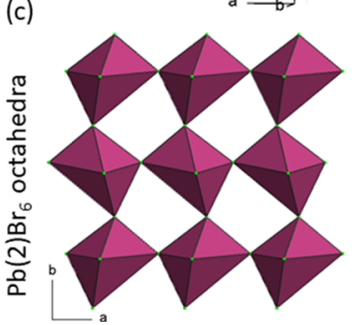

Figure 2. (a) In II, the perovskite structure is built of two nonequivalent [100] layers consisting of $\mathrm{Pb}(1) \mathrm{Br}_{6}$ and $\mathrm{Pb}(2) \mathrm{Br}_{6}$ octahedra; the $\mathrm{MHy}^{+}$cations are ordered and their arrangement strongly shapes the inorganic substructure; black dotted lines represent $\mathrm{HBs}$, red dashed lines show $\mathrm{Pb}(2)-\mathrm{NH}_{2}$ coordinate bonds; (b, c) single [100] layers where the one built of $\mathrm{Pb}(2) \mathrm{Br}_{6}$ units is highly distorted.

modification of the ideal perovskite in II. To illustrate best the magnitude of the changes taking place in the structure of II, the octahedral distortion parameters of the low-symmetry phases of $\mathrm{FAPbBr}_{3}$ and $\mathrm{MAPbBr}_{3}$ are compared with those of $\mathrm{MHyPbBr}_{3}$ in Table $\mathrm{S} 4 . \mathrm{Br}-\mathrm{Pb}-\mathrm{Br}_{\text {cis }}$ angles in $\mathrm{Pb}(2) \mathrm{Br}_{6}$ spread between 71 and $132^{\circ}$ giving octahedral angle variance $\left(\sigma^{2}\right)$ roughly ten times greater than recorded previously in the most distorted phase III of $\mathrm{MAPbBr}_{3}$; the bond length distortion $\Delta d$ is more than 50 times greater than that in $\mathrm{MAPbBr}_{3}$ and 20 times bigger than that in $\mathrm{Pb}(1) \mathrm{Br}_{6}$ layers. The same applies to $\mathrm{Pb}-\mathrm{Br}-\mathrm{Pb}$ angles, which vary from $145-$ $151^{\circ}$ in the layers consisting of $\mathrm{Pb}(2) \mathrm{Br}_{6}$ and are the smallest among the three compared structures. It is worth noting that deformations of $\mathrm{Pb}(1) \mathrm{Br}_{6}$ are similar to that found in LT phases of $\mathrm{FAPbBr}_{3}$ and $\mathrm{MAPbBr}_{3}$ compounds. The specific arrangement of the cations in II not only introduces the 
distortion of the perovskite structure but also imparts polar properties (Figure S6).

To verify the absence of an inversion center in phase II, SHG was measured. It is evident that at RT, phase II features a noncentrosymmetric lattice since it displays strong SHG activity (Figure S7). Upon heating to $420 \mathrm{~K}$, the SHG signal completely vanishes, indicating the presence of an inversion center in I. Further cooling and heating cycles consistently yield restoration and suppression of the SHG signal at $650 \mathrm{~nm}$, respectively, thus demonstrating the reversibility of the monoclinic cubic phase transition. The Kurtz-Perry powder test $^{24}$ (1300 $\mathrm{nm}$ excitation) revealed that the SHG response of the RT phase is as high as 0.18 that of KDP (Figure S8a). A separate powder test at RT with $800 \mathrm{~nm}$ beam shows that at this wavelength, the SHG activity is largely suppressed by the material self-absorption of the second-harmonic radiation ${ }^{25}$ (relative SHG intensity equal to 0.0012 that of $\mathrm{KDP}$, see Figure S8b). Interestingly, at this excitation wavelength, we have also collected strong upconverted PL, which is twophoton in nature, as confirmed by power-dependent measurements (Figure S9). The two-photon absorption (TPA) cross section, $\sigma_{2}$, quantified with the use of the solid-state twophoton excited fluorescence (SSTPEF) technique ${ }^{26}$ is equal to $1920 \mathrm{GM}$ at $800 \mathrm{~nm}$ per $\mathrm{MHyPbBr}_{3}$ structural unit. A comparison of TPA properties of $\mathrm{MHyPbBr}_{3}$ with those reported for $\mathrm{CsPbBr}_{3}$ and $\mathrm{MAPbBr}_{3}$ is provided in Table S5 in the Supporting Information.

The temperature dependence of the dielectric constant $\varepsilon^{\prime}$ and dielectric loss $\varepsilon^{\prime \prime}$ of the $\mathrm{MHyPbBr}_{3}$ single crystal shows a sudden increase near $T_{c}$ on heating (Figure 3), indicating a
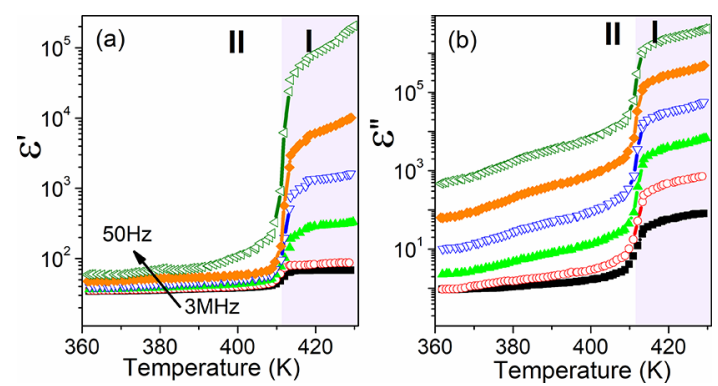

Figure 3. Temperature dependence of (a) $\varepsilon^{\prime}$ and (b) $\varepsilon^{\prime \prime}$ for the MHy $\mathrm{PbBr}_{3}$ single crystal. The representative curves are plotted in frequency decades between $50 \mathrm{~Hz}$ and $3 \mathrm{MHz}$. Marked area corresponds to phase $\mathbf{I}$.

first-order structural phase transition. This behavior resembles that observed for switchable dielectrics. ${ }^{27}$ In II, the $\varepsilon^{\prime}$ is weakly dependent on the temperature and frequency and it follows the Curie-Weiss law (Figure S10). However, it becomes strongly frequency-dependent above $T_{c}$ and increases with temperature, reaching values a few orders of magnitude higher. Such a behavior indicates the presence of electronic and/or ionic conductivity. ${ }^{28}$ The static dielectric constant, which gives the picture of the electric material polarizability in phase II for 1 $\mathrm{MHz}$, reaches a value of 35 , which is of the same order as that for $\mathrm{FAPbBr}_{3}(\sim 34)$ but higher than that reported for $\mathrm{MAPbBr}_{3}$ $(\sim 26) .{ }^{11}$ However, it should be underlined that for $\mathrm{MHyPbBr}_{3}$, this static value is reached above RT, while for other bromides, it is well below RT.

Electron-phonon interactions affect the optoelectronic properties of lead halides. To understand phonon properties, the behavior of the $\mathrm{MHy}^{+}$cation within the lead-bromide framework, and the impact of an order-disorder phenomena on the framework, temperature-dependent Raman scattering on $\mathrm{MHyPbBr}_{3}$ single crystals was performed (Figures $\mathrm{S} 11$ and S12). Vibrational wavenumbers and proposed assignment are listed in Table S6. At $320 \mathrm{~K}$, the spectra show only four intense bands below $80 \mathrm{~cm}^{-1}$ that in analogy to $\mathrm{MAPbBr}_{3}$ can be attributed to the $\mathrm{PbBr}_{6}$ librations and distortions. ${ }^{29,30}$ Weaker bands observed above $200 \mathrm{~cm}^{-1}$ correspond to internal vibrations of the $\mathrm{MHy}^{+}$cation. ${ }^{22,31}$ Temperature-dependent spectra show that the majority of bands related to $\mathrm{MHy}^{+}$ vibrations exhibit pronounced broadening on heating, indicating that the rotational freedom of the $\mathrm{MHy}^{+}$strongly increases with increasing temperature (Figures S11 and S13). Furthermore, they exhibit a sudden and very pronounced broadening at $420 \mathrm{~K}$ (Figure S13), indicating unlocking the full reorientation of the $\mathrm{MHy}^{+}$cations inside the cavities in $\mathbf{I}$. Similar behavior is observed for $\mathrm{MHy}^{+}$librational and translational modes (bands in the $300-80 \mathrm{~cm}^{-1}$ range, Figures S11 and S12a). It is worth noting that one band, observed at $315 \mathrm{~cm}^{-1}$ at $80 \mathrm{~K}$, remains very broad even at $80 \mathrm{~K}$. This band corresponds to the $\tau\left(\mathrm{CH}_{3}\right)$ mode and studies of $\mathrm{MAPbX}_{3}$ perovskites showed that this torsional mode is very sensitive to steric hindrance by the surrounding lattice cages. ${ }^{30}$ Raman spectra also show large shifts near $T_{c}$ for the $\mathrm{PbBr}_{6}$ modes (Figures S12b and S13d). This behavior proves that disordering of $\mathrm{MHy}^{+}$is associated with a pronounced change in the distortion and tilts of the $\mathrm{PbBr}_{6}$ octahedra. The modes of $\mathrm{MHy}_{\mathrm{PbBr}}$ have lower energy (the lowest energy mode at 22 $\mathrm{cm}^{-1}$ at $\left.80 \mathrm{~K}\right)$ than $\mathrm{MAPbBr}_{3}\left(39 \mathrm{~cm}^{-1}\right.$ at $\left.100 \mathrm{~K}\right){ }^{30}$ This feature suggests higher softness of $\mathrm{MHyPbBr}_{3}$. At $80 \mathrm{~K}$, Raman bands observed below $80 \mathrm{~cm}^{-1}$ (cage modes) are very narrow (full width at half-maximum (FWHM) is between 1.6 and 3.9 $\mathrm{cm}^{-1}$ ), indicating well-ordered structure and long lifetime of these phonons. Although the lowest wavenumber modes broaden with an increase of temperature, their FWHM values are still below $10 \mathrm{~cm}^{-1}$ at RT (Figures $\mathrm{S} 12$ and S13d) while such modes were very broad at $\mathrm{RT}$ for $\mathrm{MAPbX}_{3}$ perovskites. $^{29,30}$ This behavior implies significantly longer phonon lifetimes in $\mathrm{MHyPbBr}_{3}$ compared to $\mathrm{MAPbX}_{3}$. It has been argued that for $\mathrm{MAPbX}_{3}$ compounds, short phonon lifetimes contributed to the reduction of charge-carrier mobility through electron-phonon interactions. ${ }^{30}$ Thus, although the cage optical phonons of $\mathrm{MHyPbBr}_{3}$ are expected to be significantly populated at RT, their contribution to the electron scattering should be weaker than in $\mathrm{MAPbX}_{3}$ analogues.

The diffuse reflectance spectrum shows a band at $469 \mathrm{~nm}$ (Figure S14) that can be attributed to excitonic absorption. The energy band gap $E_{\mathrm{g}}(2.58 \mathrm{eV})$ is significantly larger compared to the values observed for $\mathrm{MAPbBr}_{3}$ and $\mathrm{FAPbBr}$ $(2.18-2.30 \mathrm{eV}) .^{32}$ In $3 \mathrm{D}$ lead halides, the band gap increases with increasing $\mathrm{Pb}-\mathrm{X}$ bonds and the positional distortion of $\mathrm{X}^{-}$ions from the cubic symmetry sites. ${ }^{33}$ Thus, the observed blue shift of the band gap for $\mathrm{MHyPbBr}_{3}$ is consistent with large distortion of the inorganic framework in $\mathrm{MHyPbBr}_{3}$. Temperature-dependent absorption spectra show a red shift of $E_{\mathrm{g}}$ on heating and a large jump at $T_{\mathrm{c}}$ (Figure S15). The abrupt band gap narrowing at $T_{\mathrm{c}}$ is consistent with symmetrization of the structure, that is, disappearance of the $\mathrm{PbBr}_{6}$ octahedra tilting and distortion in cubic phase I. Interestingly, the band gap further decreases with increasing temperature in $\mathbf{I}$, whereas widening of the band gap was suggested for $\mathrm{MAPbBr}_{3}$ and $\mathrm{FAPbBr}_{3}{ }^{13,32}$ The $E_{\mathrm{g}}$ of phase $\mathbf{I}$ is still significantly larger (2.41 $\mathrm{eV}$ at $435 \mathrm{~K}$ ) compared to those of $\mathrm{MAPbBr}_{3}$ and $\mathrm{FAPbBr}_{3}$. 
Thus, symmetrization of the structure cannot fully explain the relatively large band gap of $\mathrm{MHyPbBr}_{3}$.

The PL spectrum of $\mathrm{MHyPbBr}_{3}$ recorded at $80 \mathrm{~K}$ shows a very narrow band at $457.9 \mathrm{~nm}$ (FWHM, $3.9 \mathrm{~nm}$ ) and two broader bands at 485.7 (FWHM, $17.3 \mathrm{~nm}$ ) and $505.7 \mathrm{~nm}$ (FWHM, $19.5 \mathrm{~nm}$ ) (Figure 4 and Figure S16). The narrow

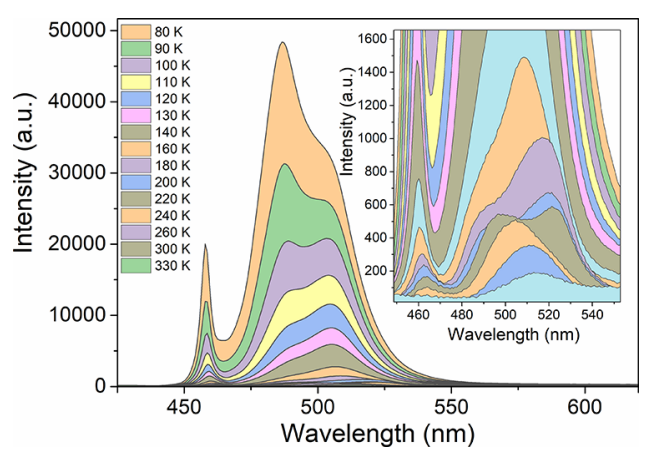

Figure 4. Temperature-dependent $\mathrm{PL}$ spectra of $\mathrm{MHyPbBr}_{3}$ measured every $10 \mathrm{~K}$ from 80 to $330 \mathrm{~K}$.

band originates from free exciton (FE) recombination, observed for $\mathrm{MAPbBr}_{3}$ and $\mathrm{FAPbBr}_{3}$ near 545-550 nm. ${ }^{32,34}$ Former studies of $\mathrm{MAPbBr}_{3}$ revealed also bands near 551 and $556 \mathrm{~nm}$ at $60 \mathrm{~K}$ that were assigned to bound excitons (BEs). ${ }^{34}$ Time-resolved measurements at $80 \mathrm{~K}$ under a $375 \mathrm{~nm}$ excitation line generated by a femtosecond laser show that the emission near $460 \mathrm{~nm}$ decays quickly $(0.156 \mathrm{~ns})$ while that at longer wavelengths relaxes more slowly $(0.700 \mathrm{~ns})$ (Figure S17). Based on literature data for $\mathrm{MAPbBr}_{3}$, large Stokes shifts and FWHM values for the 485.7 and $505.7 \mathrm{~nm}$ bands, and much longer lifetime compared to the FE emission, we assign the broader emission to BE states. It is worth noting, however, that the red shift of $\mathrm{BE}$ emission was much smaller for $\mathrm{MAPbBr}_{3}$ (up to $12 \mathrm{~nm}$ at $60 \mathrm{~K}$ ) than for $\mathrm{MHyPbBr}_{3}(47.8$ and $27.8 \mathrm{~nm}$ at $80 \mathrm{~K}$ ). Such behavior points to deeper traps in $\mathrm{MHyPbBr}_{3}$, consistent with strong distortion of the perovskite framework. The PL quantum yield of $\mathrm{MHyPbBr}_{3}$ reaches $0.5 \%$ at RT. Such a low value of PLQY at RT is typical for 3D perovskites due to small exciton binding energies. ${ }^{4}$ However, PLQY can be enhanced to nearly $100 \%$ by reducing the crystallite size. $^{35}$

A monotonic red shift of the FE band with increasing temperature (Figure S18) correlates well with the decrease of $E_{\mathrm{g}}$. Again, this behavior is different from that observed for $\mathrm{FAPbBr}_{3}$ and $\mathrm{MAPbBr}_{3}$ that showed blue shifts of $\mathrm{FE}$ on heating. ${ }^{13,32}$
The PL intensity decreases on heating (Figures 4 and 5a) and the color changes from green at $80 \mathrm{~K}$ to yellowish-green above $230 \mathrm{~K}$ (Figure $5 \mathrm{~b}$ ). To acquire the exciton binding energy, the temperature dependent integrated PL intensity of FE was fitted using the following equation:

$$
I(T)=\frac{I_{0}}{1+\mathrm{e}^{-E_{\mathrm{a}} / k_{\mathrm{B}} T}}
$$

where $I_{0}, E_{a}$ and $k_{\mathrm{B}}$ correspond to the emission intensity at low temperature, the activation energy, and the Boltzmann constant, respectively. From the fit of the LT experimental data, the exciton bonding energy of $\mathrm{MHyPbBr}_{3}$ was found to be $63 \mathrm{meV}$ (Figure S19). This value is comparable to the 53.35 $\mathrm{eV}$ value found for $\mathrm{MAPbBr}_{3}$ but significantly larger than the $21.67 \mathrm{eV}$ value reported for $\mathrm{FAPbBr}_{3}{ }^{32}$

The short discussion indicates that the optical properties of $\mathrm{MHyPbBr} r_{3}$ differ significantly from the properties of $\mathrm{MAPbBr}_{3}$ and $\mathrm{FAPbBr}_{3}$. In particular, $\mathrm{MHyPbBr}_{3}$ exhibits a significantly larger band gap and blue-shifted FE emission, which shift to higher wavelengths on heating. Such behavior is expected for quasi-2D perovskites. For instance, $(\mathrm{BA})_{2}(\mathrm{MA})_{1-n} \mathrm{~Pb}_{n} \mathrm{I}_{3 n+1}$ perovskites $(\mathrm{BA}=$ butylammonim $)$ showed an increase of $E_{\mathrm{g}}$ (PL) from $1.50 \mathrm{eV}(1.60 \mathrm{eV})$ for $\mathrm{MAPbI}_{3}$ to $1.91 \mathrm{eV}(1.90 \mathrm{eV})$ for the $n=4$ analogue $\left(\mathrm{BA}_{2} \mathrm{MA}_{3} \mathrm{~Pb}_{3} \mathrm{I}_{10}\right){ }^{36}$ However, $\mathrm{MHyPbBr}_{3}$ is a $3 \mathrm{D}$ perovskite without any organic layers separating inorganic slabs, and its band gap is significantly smaller than the $2.76 \mathrm{eV}$ value reported for quasi-2D $\mathrm{EA}_{4} \mathrm{~Pb}_{3} \mathrm{Br}_{10}(\mathrm{EA}=$ ethylammonium $){ }^{37}$ Interestingly, similar to that of $\mathrm{MHyPbBr}_{3}, \mathrm{PL}$ of $\mathrm{EA}_{4} \mathrm{~Pb}_{3} \mathrm{Br}_{10}$ also consists of a weak band near $E_{\mathrm{g}}$ and a much broader, stronger, and asymmetric band redshifted by $\sim 40 \mathrm{~nm}$. This behavior was attributed to large distortion of the inorganic layers. ${ }^{37}$ Thus, the unusual behavior of $3 \mathrm{D} \mathrm{MHyPbBr}_{3}$ can be partially attributed to the presence of two layers, one less distorted and the other strongly deformed in phase II. As a result, the electronic dispersion can be highly anisotropic. However, since the band gap of $\mathbf{I}$ is still significantly larger compared to $\mathrm{MAPbBr}_{3}$ and $\mathrm{FAPbBr}_{3}$, in spite of the same $P m \overline{3} m$ cubic structure with disordered organic cations, other factors such as different dielectric properties of $\mathrm{MHy}^{+}$compared to $\mathrm{MA}^{+}$and $\mathrm{FA}^{+}$and stronger interactions between organic and inorganic components also contribute to this behavior.

In summary, we demonstrated a new 3D perovskite embedding unprecedentedly large $\mathrm{MHy}^{+}$cations. This material shows polar order, strong SHG at RT, and complicated thermochromic behavior. $\mathrm{MHyPbBr}_{3}$ also exhibits switchable dielectric properties at $418 \mathrm{~K}$ associated with the structural phase transition. Therefore, this study paves the way for a new

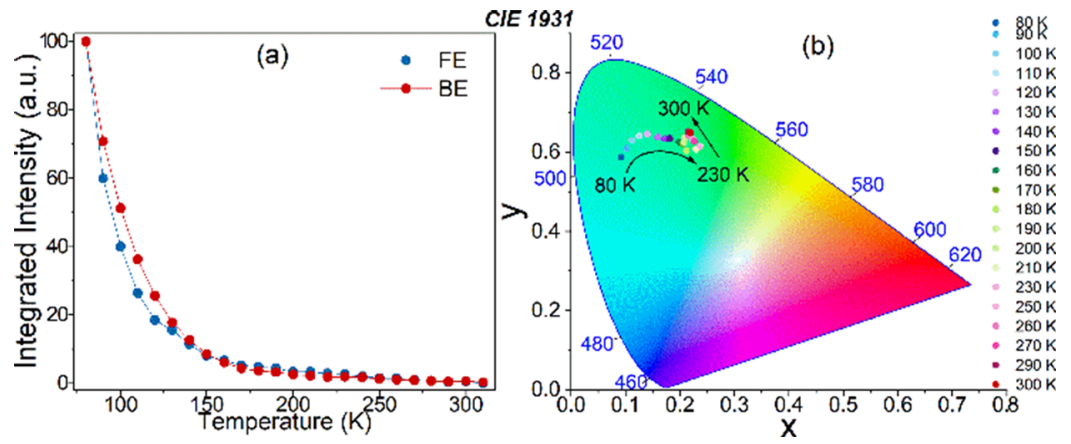

Figure 5. (a) Temperature-dependent integrated intensities for $\mathrm{FE}$ and $\mathrm{BE}$ bands, and (b) CIE coordinates of $\mathrm{MHyPbBr}_{3}$ at different temperatures. 
class of polar and light-emitting 3D perovskites with tunable properties for optoelectronic applications by synthesizing analogues with other halide anions or divalent cations as well as mixed cation or anion phases.

\section{EXPERIMENTAL SECTION}

Synthesis. $\mathrm{PbBr}_{2}$ (98\%, Sigma-Aldrich), methylhydrazine (98\%, Sigma-Aldrich), hydrobromic acid (48 wt \% in $\mathrm{H}_{2} \mathrm{O}$, Sigma-Aldrich), and methylacetate (99.5\%, Sigma-Aldrich) were commercially available and used without further purification. In order to grow single crystals, the crystal precursor was prepared by adding $5 \mathrm{mmol}$ of $\mathrm{PbBr}_{2}$ to a solution containing $5 \mathrm{mmol}$ of methylhydrazine dissolved in $5 \mathrm{~mL}$ of $\mathrm{HBr}$. The clear solution obtained after stirring for 15 min was placed into a glass vial and this vial was placed in a second larger glass vial containing methylacetate. The lid of the outer vial was thoroughly sealed whereas the lid of the inner vial was loosened to allow diffusion of methylacetate into the precursor solution. Dark yellow platelets with dimensions up to $15 \mathrm{~mm}$ were harvested after 5 days, filtered from the mother liquid, and dried at room temperature.

DSC and Thermogravimetric Analysis (TGA). Heat capacity was measured using a Mettler Toledo DSC-1 calorimeter with a high resolution of $0.4 \mu \mathrm{W}$. Nitrogen was used as a purging gas and the heating and cooling rates were $5 \mathrm{~K} / \mathrm{min}$. The sample weight was 28.55 $\mathrm{mg}$. The excess heat capacity associated with the phase transitions was evaluated by subtraction from the data, with the baseline representing variation in the absence of the phase transitions. The TGA study was performed in the temperature range $300-1180 \mathrm{~K}$ using a Perkin Elmer TGA 4000. The sample weight was $\sim 20.41 \mathrm{mg}$ and the heating speed rate was $10 \mathrm{~K} / \mathrm{min}$. Pure nitrogen gas as an atmosphere was used.

X-ray Powder Diffraction. Powder XRD pattern was obtained on an X'Pert PRO X-ray diffraction system equipped with a PIXcel ultrafast line detector and Soller slits for $\mathrm{CuK} \alpha$ radiation $(\lambda=$ $1.54056 \AA)$. The powder was measured in the reflection mode and the $\mathrm{X}$-ray tube settings were $30 \mathrm{~mA}$ and $40 \mathrm{kV}$.

Single Crystal X-ray Diffraction. For all structures, $\mathrm{CH}_{7} \mathrm{Br}_{3} \mathrm{~N}_{2} \mathrm{~Pb}, M_{\mathrm{r}}=494.01$. Single-crystal diffraction experiments were carried out with Mo $\mathrm{K} \alpha$ radiation using an Xcalibur, Atlas diffractometer. Numerical absorption correction based on Gaussian integration over a multifaceted crystal model was applied in CrysAlis PRO 1.171.38.43 (Rigaku Oxford Diffraction, 2015). Additionally, empirical absorption correction using spherical harmonics, implemented in the SCALE3 ABSPACK scaling algorithm, was employed. The structures were solved using direct methods in Shelxt and refined in Shelxl (SHELXT 2018/2, SHELXL2018/3). Hydrogen atoms were introduced from geometry and refined as riding atoms.

SHG and Two-Photon Excited Photoluminescence. SHG studies were performed using a laser system consisting of a Quantronix Integra-C regenerative amplifier operating as an $800 \mathrm{~nm}$ pump and a Quantronix-Palitra-FS BIBO crystal-based optical parametric amplifier (OPA). This system delivers wavelength-tunable pulses of $\sim 130 \mathrm{fs}$ length and operates at a repetition rate of $1 \mathrm{kHz}$. The output from the Quantronix-Palitra-FS femtosecond OPA is tunable in the range from 460 to $2000 \mathrm{~nm}$. Studies employing a 1300 $\mathrm{nm}$ wavelength have been performed using the output from the Quantronix-Palitra-FS OPA, whereas those using $800 \mathrm{~nm}$ employed direct output from a regenerative amplifier.

Assessment of SHG efficiency of $\mathrm{MHyPbBr}_{3}$ was performed with the use of the Kurtz-Perry technique. Potassium dihydrogen phosphate (KDP) was used as an SHG reference. The single crystals of $\mathrm{MHyPbBr}_{3}$ and $\mathrm{KDP}$ were crushed with a spatula and sieved through a mini-sieve set (Aldrich), collecting a microcrystal size fraction of 125-177 $\mu \mathrm{m}$. Next, size-graded samples of $\mathrm{MHyPbBr}_{3}$ and $\mathrm{KDP}$ were fixed between microscope glass slides (forming tightly packed layers), sealed, and mounted onto the sample holder. The Kurtz-Perry test was performed at $298 \mathrm{~K}$ for two wavelengths: 800 and $1300 \mathrm{~nm}$. Average powers of 800 and $1300 \mathrm{~nm}$ beams used for the Kurtz-Perry study were equal to 780 and $75 \mathrm{~mW}$, respectively.
The laser beam was directed onto samples at $45^{\circ}$ and was unfocused in all cases. A signal-collecting optics, mounted onto the glass optical fiber, was placed perpendicularly to the plane of the sample (back-scattering geometry). Scattered pumping radiation was suppressed with the use of a $700 \mathrm{~nm}$ short-pass dielectric filter (FESH0700, Thorlabs). The emission spectra were recorded using an Ocean Optics QE Pro-FL spectrograph.

The examination of the SHG response of room-temperature and high-temperature phases was conducted in a separate measurement (using $1300 \mathrm{~nm}$ beam, $75 \mathrm{~mW}$ ) in which the sample was mounted onto a thermostated heating plate (Heidolph) equipped with an external thermocouple. The sample preparation protocol was the same as for the Kurtz-Perry test.

Determination of the two-photon absorption cross section value at $800 \mathrm{~nm}$ was performed with the use of the solid-state two-photon excited fluorescence technique (SSTPEF). Prior to SSTPEF measurements, the solid samples of $\mathrm{MHyPbBr}_{3}$ and reference compound bis(4-diphenylamino)stilbene (BDPAS) were finely crushed and fixed between microscope glass slides. The two-photon upconverted emission was performed under exactly the same beam and geometrical parameters. The density of $\mathrm{MHyPbBr}_{3}\left(3.922 \mathrm{~g} \cdot \mathrm{cm}^{-3}\right)$ necessary for calculation of the two-photon absorption cross section value was taken from crystallographic data for the RT phase.

Caution! Working with the high-power laser brings danger to the eyes, especially in the spectral range in which the beam is invisible. Adequate eye protection should be used during measurements.

Dielectric Properties. The dielectric measurements were performed using a Novocontrol Alpha impedance analyzer. The dimensions of the $\mathrm{MHyPbBr}_{3}$ single crystal with crystallographic orientations perpendicular to the $(001)$ plane was $3.5 \times 2.2 \times 0.9$ $\mathrm{mm}$. Silver paste was used to ensure good electrical contact. The AC voltage with an amplitude of $1 \mathrm{~V}$ and frequency in the range $1 \mathrm{~Hz}-3$ $\mathrm{MHz}$ was applied across the sample. The temperature was controlled using a Novocontrol Quattro system by using a nitrogen gas cryostat. All dielectric measurements were taken every $1 \mathrm{~K}$ during the heating cycle. The temperature stability of the samples was better than $0.1 \mathrm{~K}$.

Raman and IR Studies. Temperature-dependent Raman spectra on a single crystal were measured using a Renishaw InVia Raman spectrometer equipped with a confocal DM 2500 Leica optical microscope, a thermoelectrically cooled CCD as a detector, and a diode laser operating at $830 \mathrm{~nm}$. Additional measurements of lowwavenumber modes (down to $5 \mathrm{~cm}^{-1}$ ) were performed on the same instrument using an eclipse filter. The temperature was controlled using a Linkam cryostat cell. The spectral resolution was $2 \mathrm{~cm}^{-1}$.

Electron Absorption, Steady-State, and Time-Resolved Photoluminescence. The RT diffuse reflectance spectrum of the powdered sample and temperature-dependent absorption spectra of a single crystal were measured using the Varian Cary 5E UV-VIS-NIR spectrophotometer. Temperature-dependent emission spectra under a $405 \mathrm{~nm}$ excitation line from a diode laser were measured with the Hamamatsu photonic multichannel analyzer PMA-12 equipped with a BT-CCD linear image sensor. The temperature of the sample was controlled using a Linkam THMS 600 heating/freezing stage. Quantum efficiency was measured on a Hamamatsu Absolute PL quantum yield measurement system C9920-02G. To record timeresolved PL spectra and decay times, a femtosecond laser (Coherent Model Libra) was used as an excitation source.

\section{ASSOCIATED CONTENT}

\section{Supporting Information}

The Supporting Information is available free of charge at https://pubs.acs.org/doi/10.1021/acs.chemmater.9b05273.

Tables S1-S6: crystal data, selected geometrical and hydrogen bond parameters, two-photon absorption cross sections, Raman wave numbers; Figures S1-S19: powder XRD pattern, DSC traces, TGA plot, histogram, polar arrangement, SHG spectra, upconverted PL, Raman spectra, diffuse reflectance and PL spectra, 
temperature-dependent absorption spectra, emission decay curves, dependence of band center position, and FWHM values for FE band and Raman bands (PDF)

CIF file for structure at RT (CIF)

CIF file for structure at $430 \mathrm{~K}$ (CIF)

\section{AUTHOR INFORMATION}

\section{Corresponding Author}

Miroslaw Mączka - Institute of Low Temperature and Structure Research, Polish Academy of Sciences, 50-950 Wrodaw, Poland; (1) orcid.org/0000-0003-2978-1093;

Email: m.maczka@int.pan.wroc.pl

\section{Authors}

Maciej Ptak - Institute of Low Temperature and Structure Research, Polish Academy of Sciences, 50-950 Wrodaw, Poland; orcid.org/0000-0002-4639-2367

Anna Gaggor - Institute of Low Temperature and Structure Research, Polish Academy of Sciences, 50-950 Wroclaw, Poland

Dagmara Stefańska - Institute of Low Temperature and Structure Research, Polish Academy of Sciences, 50-950 Wroclaw, Poland

Jan K. Zarȩba - Advanced Materials Engineering and Modeling Group, Faculty of Chemistry, Wroclaw University of Science and Technology, 50-370 Wroclaw, Poland; ㅇo orcid.org/00000001-6117-6876

Adam Sieradzki - Department of Experimental Physics, Wroclaw University of Science and Technology, 50-370 Wroclaw, Poland; (1) orcid.org/0000-0003-4136-5754

Complete contact information is available at: https://pubs.acs.org/10.1021/acs.chemmater.9b05273

\section{Author Contributions}

The manuscript was written through contributions of all authors. All authors have given approval to the final version of the manuscript.

\section{Notes}

The authors declare no competing financial interest.

\section{REFERENCES}

(1) Saparov, B.; Mitzi, D. B. Organic-Inorganic Perovskites: Structural Versatility for Functional Materials Design. Chem. Rev 2016, 116, 4558-4596.

(2) Li, W.; Wang, Z.; Deschler, F.; Gao, S.; Friend, R. H.; Cheetham, A. K. Chemically Diverse and Multifunctional Hybrid OrganicInorganic Perovskites. Nat Rev. Mater. 2017, 2, 16099.

(3) Wang, F.; Cao, Y.; Chen, C.; Chen, Q.; Wu, X.; Li, X.; Qin, T.; Huang, W. Materials Toward the Upscaling of Perovskite Solar Cells: Progress, Challenges, and Strategies. Adv. Funct. Mater. 2018, 28, 1803753.

(4) Zhao, X.; Ng, J. D. A.; Friend, R. H.; Tan, Z.-K. Opportunities and Challenges in Perovskite Light-Emitting Devices. ACS Photonics 2018, 5, 3866-3875.

(5) Cao, M.; Tian, J.; Cai, Z.; Peng, L.; Yang, L.; Wei, D. Perovskite Heterojunction Based on $\mathrm{CH}_{3} \mathrm{NH}_{3} \mathrm{PbBr}_{3}$ Single Crystal for HighSensitive Self-Powered Photodetector. Appl. Phys. Lett. 2016, 109, 233303.

(6) Chen, W.; Bhaumik, S.; Veldhuis, S. A.; Xing, G.; Xu, Q.; Grätzel, M.; Mhaisalkar, S.; Mathews, N.; Sum, T. C. Giant FivePhoton Absorption from Multidimensional Core-shell Halide Perovskite Colloidal Nanocrystals. Nat Commun. 2017, 8, 15198.

(7) D’Annibale, A.; Panetta, R.; Tarquini, O.; Colapietro, M.; Quaranta, S.; Cassetta, A.; Barba, L.; Chita, G.; Latini, A. Synthesis, Physico-Chemical Characterization and Structure of the Elusive
Hydroxylammonium Lead Iodide Perovskite $\mathrm{NH}_{3} \mathrm{OHPbI}_{3}$. Dalton Trans. 2019, 48, 5397-5407.

(8) Campbell, E. V.; Dick, B.; Rheingold, A. L.; Zhang, C.; Liu, X.; Vardeny, Z. V.; Miller, J. S. Structures of a Complex Hydrazinium Lead Iodide, $\left(\mathrm{N}_{2} \mathrm{H}_{5}\right)_{15} \mathrm{~Pb}_{3} \mathrm{I}_{21}$, Possessing $\left[\mathrm{Pb}_{2} \mathrm{I}_{9}\right]_{5}{ }^{-},\left[\mathrm{PbI}_{6}\right]_{4}^{-}$, and $\mathrm{I}^{-}$ Ions and $\alpha$ - and $\beta$ - $\left(\mathrm{N}_{2} \mathrm{H}_{5}\right) \mathrm{PbI}_{3}$. Chem. - Eur. J. 2018, 24, 222-229.

(9) Tian, J.; Cordes, D. B.; Quarti, C.; Beljonne, D.; Slawin, A. M. Z.; Zysman-Colman, E.; Morrison, F. D. Stable 6H OrganicInorganic Hybrid Lead Perovskite and Competitive Formation of $6 \mathrm{H}$ and 3C Perovskite Structure with Mixed A Cations. ACS Appl Energy Mater. 2019, 2, 5427-5437.

(10) Seth, C.; Khushalani, D. Non-Perovskite Hybrid Material, Imidazolium Lead Iodide, with Enhanced Stability. ChemNanoMat 2019, 5, 85-91.

(11) Govinda, S.; Kore, B. P.; Swain, D.; Hossain, A.; De, C.; Guru Row, T. N.; Sarma, D. D. Critical Comparison of $\mathrm{FAPbX}_{3}$ and $\mathrm{MAPbX}_{3}(\mathrm{X}=\mathrm{Br}$ and $\mathrm{Cl})$ : How Do They Differ? J. Phys. Chem. C 2017, 122, 13758-13766.

(12) Fabini, D. H.; Stoumpos, C. C.; Laurita, G.; Kaltzoglou, A.; Kontos, A. G.; Falaras, P.; Kanatzidis, M. G.; Seshadri, R. Reentrant Structural and Optical Properties and Large Positive Thermal Expansion in Perovskite Formamidinium Lead Iodide. Angew. Chem., Int. Ed. 2016, 55, 15392-15396.

(13) Yang, B.; Ming, W.; Du, M.-H.; Keum, J. K.; Puretzky, A. A.; Rouleau, C. M.; Huang, J.; Geohegan, D. B.; Wang, X.; Xiao, K. RealTime Observation of Order-Disorder Transformation of Organic Cations Induced Phase Transition and Anomalous Photoluminescence in Hybrid Perovskites. Adv. Mater. 2018, 30, 1705801.

(14) Liu, S.; Zheng, F.; Koocher, N. Z.; Takenaka, H.; Wang, F.; Rappe, A. M. Ferroelectric Domain Wall Induced Band Gap Reduction and Charge Separation in Organometal Halide Perovskites. J. Phys. Chem. Lett. 2015, 6, 693-699.

(15) Stoumpos, C. C.; Malliakas, C. D.; Peters, J. A.; Liu, Z.; Sebastian, M.; Im, J.; Chasapis, T. C.; Wibowo, A. C.; Chung, D. Y.; Freeman, A. J.; Wessels, B. W.; Kanatzidis, M. G. Crystal Growth of the Perovskite Semiconductor $\mathrm{CsPbBr}_{3}$ : A New Material for HighEnergy Radiation Detection. Cryst. Growth Des. 2013, 13, 2722-2727.

(16) Rakita, Y.; Meirzadeh, E.; Bendikov, T.; Kalchenko, V.; Lubomirsky, I.; Hodes, G.; Ehre, D.; Cahen, D. $\mathrm{CH}_{3} \mathrm{NH}_{3} \mathrm{PbBr}_{3}$ is not Pyroelectric Excluding Ferroelectric-Enhanced Photovoltaic Performance. APL Mater. 2016, 4, No. 051101.

(17) Schueller, E. C.; Laurita, G.; Fabini, D. H.; Stoumpos, C. C.; Kanatzidis, M. G.; Seshadri, R. Crystal Structure Evolution and Notable Thermal Expansion in Hybrid Perovskites Formamidinium Tin Iodide and Formamidinium Lead Bromide. Inorg. Chem. 2018, $57,695-701$.

(18) Garten, L. M.; Moore, D. T.; Nanayakkara, S. U.; Dwaraknath, S.; Schulz, P.; Wands, J.; Rockett, A.; Newell, B.; Persson, K. A.; Trolier-McKinstry, S.; Ginley, D. S. The Existence and Impact of Persistent Ferroelectric Domains in $\mathrm{MAPbI}_{3}$. Sci. Adv. 2019, 5, No. e9311.

(19) Rakita, Y.; Bar-Elli, O.; Meirzadeh, E.; Kaslasi, H.; Peleg, Y.; Hodes, G.; Lubomirsky, I.; Oron, D.; Ehre, D.; Cahen, D. Tetragonal $\mathrm{CH}_{3} \mathrm{NH}_{3} \mathrm{PbI}_{3}$ is Ferroelectric. Proc. Natl. Acad. Sci. U. S. A. 2017, 114, E5504-E5512.

(20) Simènas, M.; Balčiūnas, S.; Mączka, M.; Banys, J.; Tornau, E. E. Exploring the Antipolar Nature of Methylammonium Lead Halides: A Monte Carlo and Pyrocurrent Study. J. Phys. Chem. Lett. 2017, 8, 4906-4911.

(21) Gómez, A.; Wang, Q.; Goñi, A. R.; Campoy-Quiles, M.; Abate, A. Ferroelectricity-free Lead Halide Perovskites. Energy Environ. Sci. 2019, 12, 2537-2547.

(22) Mączka, M.; Ga̧gor, A.; Ptak, M.; Paraguassu, W.; Da Silva, T. A.; Sieradzki, A.; Pikul, A. Phase Transitions and Coexistence of Magnetic and Electric Orders in the Methylhydrazinium Metal Formate Frameworks. Chem. Mater. 2017, 29, 2264-2275.

(23) Glazer, A. M. The Classification of Tilted Octahedra in Perovskites. Acta Crystallogr. B 1972, 28, 3384-3392. 
(24) Kurtz, S. K.; Perry, T. T. A Powder Technique for the Evaluation of Nonlinear Optical Materials. J. Appl. Phys. 1968, 39, 3798-3813.

(25) Zaręba, J. K.; Białek, M. J.; Janczak, J.; Nyk, M.; Zoń, J.; Samoć, M. Beyond Single-Wavelength Shg Measurements: SpectrallyResolved Shg Studies of Tetraphosphonate Ester Coordination Polymers. Inorg. Chem. 2015, 54, 10568-10575.

(26) Medishetty, R.; Zarçba, J. K.; Mayer, D.; Samoć, M.; Fischer, R. A. Nonlinear Optical Properties, Upconversion and Lasing in MetalOrganic Frameworks. Chem. Soc. Rev. 2017, 46, 4976-5004.

(27) Tao, K.; Han, S.; Ji, C.; Liu, X.; Wu, Z.; Zhang, J.; Luo, J.; Sun, Z. Structural Phase Transition and Switchable Dielectric Properties of a Unique Two-Dimensional Organic-Inorganic Hybrid Perovskite Compound $\left[\mathrm{C}_{6} \mathrm{H}_{11} \mathrm{NH}_{2} \mathrm{CH}_{3}\right]_{4} \mathrm{~Pb}_{3} \mathrm{I}_{10}$. Cryst. Growth Des. 2018, 18, $7316-7322$.

(28) García-Fernández, A.; Bermúdez-García, J. M.; Castro-García, S.; Llamas-Saiz, A. L.; Artiaga, R.; López-Beceiro, J.; Hu, S.; Ren, W.; Stroppa, A.; Sánchez-Andújar, M.; Señarís-Rodríguez, M. A. Phase Transition, Dielectric Properties, and Ionic Transport in the $\left[\left(\mathrm{CH}_{3}\right)_{2} \mathrm{NH}_{2}\right] \mathrm{PbI}_{3}$ Organic-Inorganic Hybrid with $2 \mathrm{H}$-Hexagonal Perovskite Structure. Inorg. Chem. 2017, 56, 4918-4927.

(29) Yaffe, O.; Guo, Y.; Tan, L. Z.; Egger, D. A.; Hull, T.; Stoumpos, C. C.; Zheng, F.; Heinz, T. F.; Kronik, L.; Kanatzidis, M. G.; Owen, J. S.; Rappe, A. M.; Pimenta, M. A.; Brus, L. E. Local Polar Fluctuations in Lead Halide Perovskite Crystals. Phys. Rev. Lett. 2017, 118, 136001.

(30) Leguy, A. M. A.; Goñi, A. R.; Frost, J. M.; Skelton, J.; Brivio, F.; Rodríguez-Martínez, X.; Weber, O. J.; Pallipurath, A.; Alonso, M. I.; Campoy-Quiles, M.; Weller, M. T.; Nelson, J.; Walsh, A.; Barnes, P. R. F. Dynamic Disorder, Phonon Lifetimes, and the Assignment of Modes to the Vibrational Spectra of Methylammonium Lead Halide Perovskites. Phys. Chem. Chem. Phys. 2016, 18, 27051-27066.

(31) Mączka, M.; Ptak, M.; Ga̧gor, A.; Stefańska, D.; Sieradzki, A. Layered Lead Iodide of [Methylhydrazinium] $]_{2} \mathrm{PbI}_{4}$ with a Reduced Band Gap: Thermochromic Luminescence and Switchable Dielectric Properties Triggered by Structural Phase Transitions. Chem. Mater. 2019, 31, 8563-8575.

(32) Dai, J.; Zheng, H.; Zhu, C.; Lu, J.; Xu, C. Comparative Investigation on Temperature-dependent Photoluminescence of $\mathrm{CH}_{3} \mathrm{NH}_{3} \mathrm{PbBr}_{3}$ and $\mathrm{CH}\left(\mathrm{NH}_{2}\right)_{2} \mathrm{PbBr}_{3}$ microstructures. J. Mater. Chem. C 2016, 4, 4408-4413.

(33) Kim, J.; Lee, S.-C.; Lee, S.-H.; Hong, K.-H. Importance of Orbital Interactions in Determining Electronic Band Structures of Organo-Lead Iodide. J. Phys. Chem. C 2015, 119, 4627-4634.

(34) Chen, C.; Hu, X.; Lu, W.; Chang, S.; Shi, L.; Li, L.; Zhong, H.; Han, J.-B. Elucidating the phase transitions and temperaturedependent photoluminescence of $\mathrm{MAPbBr}_{3}$ single crystal. J. Phys. D: Appl. Phys. 2018, 51, No. 045105.

(35) Li, F.; Yang, L.; Cai, Z.; Wei, K.; Lin, F.; You, J.; Jiang, T.; Wang, Y.; Chen, X. Enhancing Exciton Binding Energy and Photoluminescence of Formamidinium Lead Bromide by Reducing Its Dimensions to 2D Nanoplates for Producing Efficient Light Emitting Diodes. Nanoscale 2018, 10, 20611-20617.

(36) Stoumpos, C. C.; Cao, D. H.; Clark, D. J.; Young, J.; Rondinelli, J. M.; Jang, J. I.; Hupp, J. T.; Kanatzidis, M. G. Ruddlesden-Popper Hybrid Lead Iodide Perovskite 2D Homologous Semiconductors. Chem. Mater. 2016, 28, 2852-2867.

(37) Mao, L.; Wu, Y.; Stoumpos, C. C.; Traore, B.; Katan, C.; Even, J.; Wasielewski, M. R.; Kanatzidis, M. G. Tunable White-Light Emission in Single-Cation-Templated Three-Layered 2D Perovskites $\left(\mathrm{CH}_{3} \mathrm{CH}_{2} \mathrm{NH}_{3}\right)_{4} \mathrm{~Pb}_{3} \mathrm{Br}_{10-\mathrm{x}} \mathrm{Cl}_{\mathrm{x}}$. J. Am. Chem. Soc. 2017, 139, 1195611963. 